\title{
Erratum to: LRRK2 G2385R variant carriers of female Parkinson's disease are more susceptible to motor fluctuation
}

\author{
Chao Gao · Hao Pang • Xiao-Guang Luo • \\ Yan Ren $\cdot$ Hong Shang $\cdot$ Zhi-Yi He
}

Published online: 20 October 2013

(C) Springer-Verlag Berlin Heidelberg 2013

\section{Erratum to: J Neurol}

DOI 10.1007/s00415-013-7086-9

The original version of this article unfortunately contained a mistake. The affiliation of the authors Chao Gao, XiaoGuang Luo, Yan Ren and Zhi-Yi He is incorrect. The correct affiliation should be:

Neurology Department of First Affiliated Hospital of China Medical University, Shenyang, People's Republic of China.

The online version of the original article can be found under doi:10.1007/s00415-013-7086-9.

C. Gao · X.-G. Luo $(\bowtie) \cdot$ Y. Ren $\cdot$ Z.-Y. He Neurology Department of First Affiliated Hospital of China Medical University, Shenyang, People's Republic of China

e-mail: grace_shenyang@163.com

\section{H. Pang}

Department of Forensic Serology, China Medical University,

Shenyang, People's Republic of China

\section{X.-G. Luo $\cdot$ H. Shang}

Department of Laboratory Medicine, First Affiliated Hospital of China Medical University, 155\# Nanjing Bei Street, Heping

District, Shenyang 110001, People's Republic of China 